Review Article

\title{
Effect and Molecular Mechanisms of Traditional Chinese Medicine on Regulating Tumor Immunosuppressive Microenvironment
}

\author{
Qiujun Guo, ${ }^{1,2}$ Jie Li, ${ }^{1}$ and Hongsheng Lin ${ }^{1}$ \\ ${ }^{1}$ Department of Oncology, Guang'anmen Hospital, China Academy of Chinese Medical Sciences, No. 5 Beixiange, \\ Xicheng District, Beijing 100053, China \\ ${ }^{2}$ Beijing University of Chinese Medicine, No. 11 North Third Ring Road East, Chaoyang District, Beijing 100029, China
}

Correspondence should be addressed to Jie Li; drjieli2007@126.com

Received 24 July 2014; Accepted 16 December 2014

Academic Editor: Yung-Fu Chang

Copyright (C) 2015 Qiujun Guo et al. This is an open access article distributed under the Creative Commons Attribution License, which permits unrestricted use, distribution, and reproduction in any medium, provided the original work is properly cited.

\begin{abstract}
Traditional Chinese medicine (TCM) is an important complementary strategy for treating cancer in China. The mechanism is related to regulating the internal environment and remodeling the tumor immunosuppressive microenvironment (TIM). Herein we illustrate how TIM is reformed and its protumor activity on promoting tumor cell proliferation, angiogenesis and lymphangiogenesis, tumor invasion, and the oncogenicity of cancer stem cells. Furthermore we summarize the effects and mechanism of TCM on regulating TIM via enhancing antitumor immune responses (e.g., regulating the expression of MHC molecules and Fas/FasL, attenuating cancerigenic ability of cancer stem cells) and remolding immunosuppressive cells (e.g., reversing immune phenotypes of T lymphocytes and tumor associated macrophages, promoting dendritic cells mature, restraining myeloid derived suppressor cells function, and regulating Th1/Th2 factors). We also reveal the bidirectional and multitargeting functions of TCM on regulating TIM. Hopefully, it provides new theoretical basis for TCM clinical practice in cancer treatment and prevention.
\end{abstract}

\section{Introduction}

Chronic inflammation and immune suppression are the two core characteristics of the tumor microenvironment. It has been proven that chronic inflammation plays an important role in tumorigenesis and development; for instance inflammatory large intestine disease leads to colorectal cancer, $\mathrm{H}$. Pylori infection breeds gastric cancer, and hepatitis B and $\mathrm{C}$ virus infection causes hepatocellular carcinoma (HCC). However, immune cells in tumor microenvironment promote tumor progression on the other hand: they constitute tumor immunosuppressive microenvironment (TIM) and alleviate tumor immune escape and tumorigenesis. Harmful stimulating factors such as hypoxia, acid environment, hyperosmosis, and inflammatory cytokines in tumor microenvironment facilitate the formation of TIM. According to the theory of tumor immunoediting, in TIM released tumor cells and immunosuppressive factors remodel the phenotype of immune cells, which decreases its antitumor function; meanwhile, remodeled immune cells "resculpture" tumor cells and make them become of low immunogenicity and might favor immune escape of tumor cells $[1,2]$. Besides these, immune suppressive cells in TIM also bring out angiogenesis and lymphangiogenesis, playing a vital role in tumor development and metastasis. Thus, TIM is considered as a novel target for cancer treatment.

Traditional Chinese medicine (TCM) is a very important tumor treatment strategy in China [3]. It is accepted that TCM can reduce the toxicity of chemotherapy and radiotherapy, enhance the antitumor effect of these therapies, alleviate tumor-induced clinical symptoms and cancer pains, and prolong the survival time of postoperational and advanced stage cancer patients [4]. Though the effect mechanism of TCM is not very clear, increasing data has shown that it may relate 
with its action on regulating tumor immune environment, the novel target according to the theory of immunoediting $[5,6]$. In this paper, we will introduce the formation and function of TIM and summarize recent researches of TCM on regulating it.

\section{Tumor and Its Matrix Cells Constitute Tumor Immunosuppressive Microenvironment}

Tumor microenvironment is a complex compound, including tumor cells, stromal cells, extracellular matrix, cellular factors, and chemokines. The microenvironment plays a pivotal role in the process of cancer development and metastasis. Downregulating the immune function and combined with extracellular matrix proteins and matrix-degrading enzymes, stromal cells (e.g., angiogenic cells, immune cells, and tumor associated fibroblasts) and cell factors they produced encompass tumor cells and form the tumor immunosuppressive microenvironment [7].

In TIM, tumor cells do not express classical MHC I molecules but express nonclassical MHC molecules, leading to the immunosuppression and tumor progression [8]. Meanwhile, tumor cells express a variety of immunosuppressive factors, such as IL-10, IL-6, and TGF- $\beta$, reversing immune cells to immunosuppressive phenotypes, for example, regulatory $\mathrm{T}$ cells, regulatory B cells, tumor associated macrophages (TAMs), regulatory dendritic cells, and myeloid derived suppressor cells (MDSCs). These immunosuppressive cells interact with each other and increasingly proliferate and express immunosuppressive and protumor factors. Moreover, they consume proinflammatory factors (IL-2, TNF$\alpha$ ), promote $\mathrm{T}$ cells apoptosis, and reduce the antitumor activity of NK cells. They produce matrix metalloproteinases (MMPs) and vascular endothelial growth factors (VEGF) as well, promoting angiogenesis and tumor invasion [9-12]. All these tumorigenesis responses give negative feedback to immunocytes, breeding a vicious circle for tumor treating.

\section{Immune Suppressive Cells in TIM Promote Tumorigenesis and Progression}

3.1. Promoting Tumor Cell Proliferation. Studies have proven the close relationship between TIM and tumorigenesis in different kinds of cancer diseases [13, 14]. When turning to basic researches, Fritz et al. [15] found that macrophages produce IGF-1 which directly stimulates neoplastic proliferation through Erk and Akt activation. Munari et al. [16] demonstrated that gastric lymphoma-infiltrating macrophages highly produced APRIL, which was regarded as a novel cytokine crucial in sustaining B cell proliferation and causing a mucosa associated lymphoid tissue B cell lymphoma proliferation. When coculturing with breast cancer cells, IL-4-activated macrophage transported microRNAs from itself to breast cancer cells, and one of microRNAs, miR-223, promoted the invasion of breast cancer cells via the Mef2c- $\beta$-catenin pathway [17]. These studies conclude and inside provide evidences that immune suppressive cells in TIM promote tumor cells multiplication.

3.2. Promoting Angiogenesis and Lymphangiogenesis. Tumor hematal and lymphatic vessels are important in tumor progression and serve as paths and tubes for nutrients and metabolites transportation, promoting tumor cells growth and metastasis. TIM plays a vital role in the process of angiogenesis and lymphangiogenesis. In tumor hypoxic microenvironment, tumor cells, tumor associated macrophages, dendritic cells, myeloid derived suppressor cells, and neutrophil cells secrete a variety of proangiogenesis and prolymphangiogenesis factors (e.g., VEGF, VEGF-C, MMP-9, TGF- $\beta$, and COX-2) $[18,19]$. In recent years, studies also found a subtype of TAMs, called TIE2-expressing macrophages (TEM). TEMs overexpress angiopoietin-2 (ANG2) and mediate a crosstalk with vascular endothelial cells via Ang2-TIE2 pathway to promote angiogenesis [20]. Moreover, TAMs could transdifferentiate to lymphatic endothelial cells and, as endothelial progenitor cells, take part in lymphangiogenesis in tumor tissues under certain conditions [21]. Thereby, TIM is directly involved in the formation of vessels in tumors.

3.3. Promoting Tumor Invasion. The invasive ability of tumor cells is the basis of tumor local invasion and distant metastasis, and prior to this is the remolding of the extracellular matrix. TAM, Th-17 cells, and DCs in TIM produce MMPs and TGF- $\beta$ to dissolve extracellular collagens, so as to remold the extracellular matrix. TAMs used both the mesenchymal mode requiring MMPs and the amoeboid migration mode to infiltrate tumor cell spheroids and promote MMPindependent invasion by tumor cells [22]. Epithelial mesenchymal transition (EMT) is the morphological change process of tumor cells invasion and metastasis [23]. TAMs could mediate EMT of tumor cells and promote the tumor progression through the TLR4/IL-10 signaling pathway [24]. By activating TGF- $\beta$, EGF, and HGF signaling pathway, MDSCs induced EMT as well [25]. Hence, TIM makes it easier for tumor invasion via both affecting it straightly and mediating EMT.

3.4. Promoting the Oncogenicity of Cancer Stem Cell. Cancer stem-like cell (CSC) is a hotspot of the tumor research in recent years. It is widely thought that CSC is a tumorigenesis and metastasis "seed cell" and the "springhead" of tumor immune escape. CSC has a continuous self-renewal and proliferation ability, and it differentiates incompletely, resisting radiation and chemotherapy and producing immune suppressive factors. Losing normal exogenous apoptosis signaling pathways, CSC strongly shows the resistance to apoptosis functions [26, 27]. Immune suppressive cells in TIM also participate in regulating CSC function. Yang et al. [28] found that TAMs mediate tumorigenesis through regulation of breast CSCs and promote CSC-like phenotypes in murine breast cancer cells by upregulating their expression of Sox-2. TAM markers are associated with cancer stem cell marker in oral squamous cell carcinoma [29]. MDSCs had similar features that triggered microRNA101 expression in 


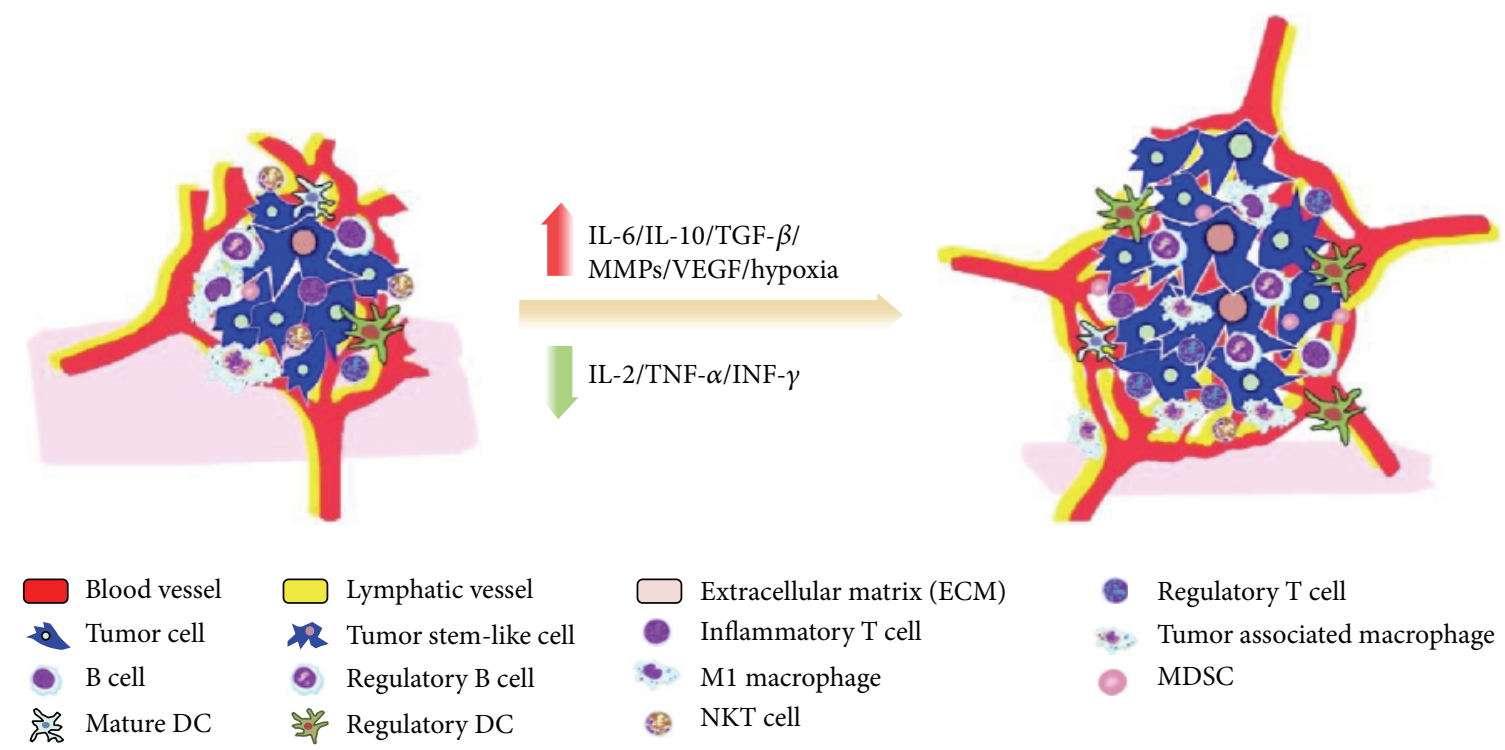

FIGURE 1: Tumor immunosuppressive microenvironment promotes tumorigenesis and progression.

cancer cells and increased cancer cell stemness and increasing metastatic and tumorigenic potential targeted stem cell core genes [30]. In conclusion, TIM enhances CSC progression in mutual effect (Figure 1).

The hypoxia environment brings TIM increasingly producing immune suppressive and angiogenesis factors (e.g., IL-6, IL-10, TGF- $\beta$, MMPs, and VEGF) and downregulating inflammatory cytokines such as IL-2, TNF- $\alpha$, and INFgamma, promoting immune suppressive cells proliferation and CSCs growth and inhibiting antitumor cells. Thus, TIM leads to tumorigenesis, angiogenesis, lymphangiogenesis, and tumor ECM remolding and causes tumor progression and metastasis.

\section{TCM Enhances Tumor Immune Responses}

4.1. TCM Promotes Classic MHC Molecules Expression. MHC, also called human leukocyte antigen (HLA) in human tissues, is the important immunological recognition molecule in the process of tumor immune response. Classic $\mathrm{MHC}$ molecule can be divided into two subgroups: MHC class I and MHC class II. MHC I have a vital role in presenting tumor antigens to $\mathrm{T}$ cell receptor. After identifying the tumor antigen peptide and MHC class I molecule, the cytotoxic $\mathrm{T}$ cell (CTL) is activated and launched a series of cytolysis reactions to kill tumor cells. MHC II presents tumor antigens to $\mathrm{CD} 4^{+} \mathrm{T}$ helper cells, leading to cellular mediated immune response. However, both immune and malignant cells in the tumor microenvironment do not express typical MHC molecules. They downregulate MHC class I expression, overexpress nonclassical HLA such as HLA-G, HLA-E, and HLA$\mathrm{F}$, which have been recently proven to be correlated with poor clinical outcome, and escape from $\mathrm{T}$ and $\mathrm{NK}$ cell-mediated recognition $[8,31]$.
Studies have shown that TCM can upregulate MHC molecules in tumor microenvironment. Li et al. [32] explored the effect of Invigorating Spleen and Detoxification Decoction (ISD) (Radix Codonopsis, Poria, Rhizoma Atractylodis Macrocephalae, Radix Glycyrrhizae, Radix Bupleuri, Rhizoma Curcumae, and Herba Scutellariae barbatae) on MHC molecules in the rat liver cancer tissue and found that ISD could enhance the expression of MHC I and MHC II and prolong the rat survival time. Besides the effects on tumor cells, TCM could also increase MHC expression in immune cells. Anoectochilus formosanus is a medicinal herb in Asia and extracts of $A$. formosanus have been reported to possess antitumor activities. Kuan et al. [33] found that $A$. formosanus could stimulate the MHC II expression. In vitro experiment showed Fei Liu Ping Extractum (FLP) (Radix Panacis Quinquefolii, Cordyceps, Radix Astragali, Radix Codonopsis, Radix Glehniae, Radix Ophiopogonis, Herba Agrimoniae, Polygonum bistorta L., Thlaspi arvense Linn., Hedyotis diffusa, Semen Juglandis, Semen Armeniacae amarum, and Bulbus Fritillariae Cirrhosae) could upregulate the expression of MHC II of dendritic cells and improve the body function of antitumor immunological surveillance [34]. These results suggest that TCM could enhance tumor antigen-presenting ability by upregulating classic MHC expression in both tumor and immune cells.

4.2. TCM Induces Tumor Cells Apoptosis via Fas/FasL Pathway. Fas and its natural ligand FasL are molecules expressed on cellular membranes. The activation of Fas/FasL pathway plays an important role in cell apoptosis. Physiologically, cytotoxic $\mathrm{T}$ lymphocytes (Fas ${ }^{\text {low }} \mathrm{FasL}^{\text {high }}$ ) express FasL and combine with the Fas expressed by target cells (Fas ${ }^{\text {high }} \mathrm{FasL}^{\text {low }}$ ), resulting in the trimerization and activation of the Fas receptor, and then mediate target cells apoptosis. 
Unlike normal target cells, tumor cells express nonfunctional Fas or low quantity of Fas; meanwhile overexpression of FasL promotes tumor cells immune escape by preventing the combination with cytotoxic $\mathrm{T}$ cells and induces CTL apoptosis [35].

Our previous study showed that TCM herbal medicine Yang Wei Kang Liu Granule (YWKL) (Radix Astragali, Radix Ginseng, Hedyotis diffusa, Yunnan Manyleaf Paris Rhizome, Radix Notoginseng, Radix Paeoniae Rubra, and Hematoxylon) could increase FasL expression and downregulate Fas expression in T lymphocytes of gastric cancer patients, which indicates YWKLF may inhibit cancer by inducing apoptosis [36]. Our subsequent studies established MGC-803 stomach cancer cell model in vitro and showed FasL mRNA in MGC803 declined significantly after treatment by YWKL [37]. These mean YWKL may enhance cancer cells' sensitivity to immune response cells like CTL and lead to tumor cells apoptosis by regulating Fas/FasL pathway.

4.3. TCM Attenuates Oncogenicity of CSCs. Cancer stemlike cells (CSCs) are a more malignant composition in tumor tissues inhibiting immune response. Researches have demonstrated CSCs take part in carcinogenesis and therapy resistance in kinds of tumors [38-41]. CSCs rarely express immune recognition molecules such as HLA-DR and costimulatory molecules such as CD80 and CD86. Furthermore, laboratory researches show CSC tumor spheres lowly express Fas and highly express membrane complement regulatory proteins and FoxP3 [42]. All these findings indicate CSCs prevent antitumor immune responses and promote immunosuppressive microenvironment formation. Thus, it is rational to target CSCs to reverse tumor immune suppression.

Some medicinal herbs have been proved to have anti-CSC ability. Chang et al. [43] cultured the hMG63-derived cancer stem cells in permissive microenvironments for stem cell differentiation and found Bufalin inhibited the proliferation and sphere formation of cancer stem cells. Zhang et al. [44] found that Huaier (Trametes robiniophila Murr.) aqueous extract had significant efficacy on inhibiting spheroid formation $(P<0.05)$ and reduced the aldehyde dehydrogenase $(\mathrm{ALDH})$ positive cell population in colorectal primary cancer cells $(P<0.05)$. Further study revealed Huaier extract downregulated the $\mathrm{Wnt} / \beta$-catenin pathway, which is one of the critical pathways demonstrated to mediate the selfrenewal of CSCs. Honokiol is an extract from medicinal herb Cortex Magnoliae Officinalis. Research showed that it can thwart tumor growth [45]. Recently, Ponnurangam et al. [46] found the ability of Honokiol to enhance the sensitivity of colon CSCs to ionizing radiation is by inhibiting the $\gamma$ secretase complex and the Notch signaling pathway. These findings indicate that Chinese medicine may reduce CSCs and weaken tumor immune resistance as well (Figure 2).

While being treated by Chinese medicine, the percentage of CSCs is decreased; Classic MHC and Fas molecules are expressed more on tumor cytomembranes, which lead to tumor cells having lower malignant degree and easier to be recognized and killed by the immune system.

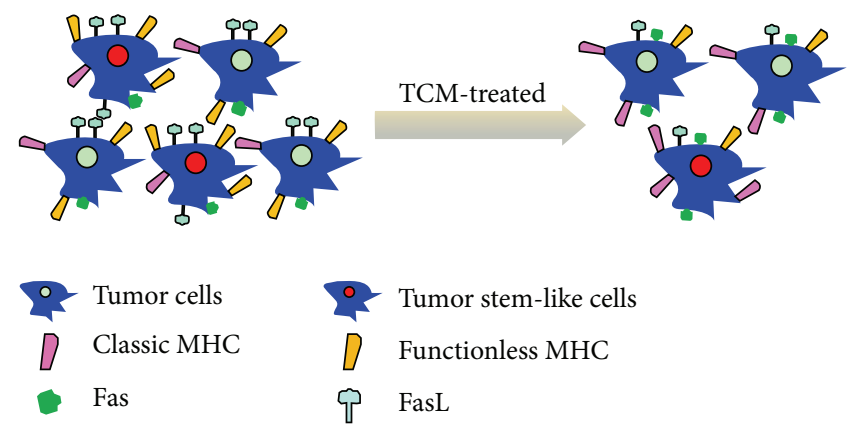

FIGURE 2: TCM enhances tumor cells immune responses.

\section{TCM Reverses the Immunosuppressive Phenotype and Regulates the Antitumor Functions of Immune Cells}

Immune cells show various functions and phenotypes and play multiple roles in antitumor immunity and protumorigenesis, which are determined and influenced by tumor immune microenvironment. TCM herbs indicate a biphasic regulation on tumor cells phenotype to enhance antitumor immune responses: increasing proinflammatory phenotypic antitumor activity while reversing and remolding the suppressive function (phenotype) of immunocytes (Figure 3).

5.1. TCM Enhances T lymphocyte Antitumor Abilities. T lymphocytes are notably involved in tumor adaptive immune cells but also have been found participating in tumor immune suppression in different subtypes [47]. T cells express specific recognition and signal transduction related to TCR/CD3 complex molecules. Based on their function and phenotypes, $\mathrm{T}$ cells are divided into three main subtypes: cytotoxic $\mathrm{T}$ cells $\left(\mathrm{CD}^{+} \mathrm{CD}^{+}\right)$, helper $\mathrm{T}$ cells $\left(\mathrm{CD}^{+} \mathrm{CD}^{+}\right)$, and regulatory $\mathrm{T}$ cells $\left(\mathrm{CD} 3^{+} \mathrm{CD} 4^{+} \mathrm{CD} 25^{+}\right) . \mathrm{CD}^{+} \mathrm{T}$ cells produce IL- 2 and IL15 which enhance the activation of $\mathrm{CD}^{+} \mathrm{T}$ cells, maintain the activity of CTL, and also activate innate immune cells such as natural kill cells and dendritic cells. Meanwhile, part of $\mathrm{CD}^{+} \mathrm{T}$ cells has the ability to kill tumor cells directly. $\mathrm{CD}^{+} \mathrm{T}$ cells release perforin and particle enzymes to kill and dissolve tumor cells and lead to target cells apoptosis through Fas/FasL pathway. $\mathrm{CD} 4^{+} \mathrm{CD} 25^{+} \mathrm{T}$ cell (Treg) is an immune regulating subtype which is regarded to play immunosuppressive role in tumor microenvironment. Tregs produce quantity of immune suppressive factors, for example, IL-10 and TGF- $\beta$, compete with response $\mathrm{T}$ cells for consuming IL-2, affect metabolism of other T cells, and downregulate the stimulation of dendritic cells [48]. Thus, it is the key to regulating tumor immune microenvironment via enhancing antitumor efficacy of T cells and inhibiting Treg cell and its function.

It is notable that TCM may adjust immune function by targeting $\mathrm{T}$ cells. Feiyanning Decoction (FYN) (Radix Astragali, Rhizoma Atractylodis Macrocephalae, Pseudobulbus Cremastrae seu Pleiones, Salvia Chinensis Benth., Paris polyphylla, Corium Bufonis, Nidus Vespae, Rhizoma Polygonati, Fructus Corni, Herba Epimedii, Fructus Ligustri Lucidi, 
Immune cells and cytokines in TIM

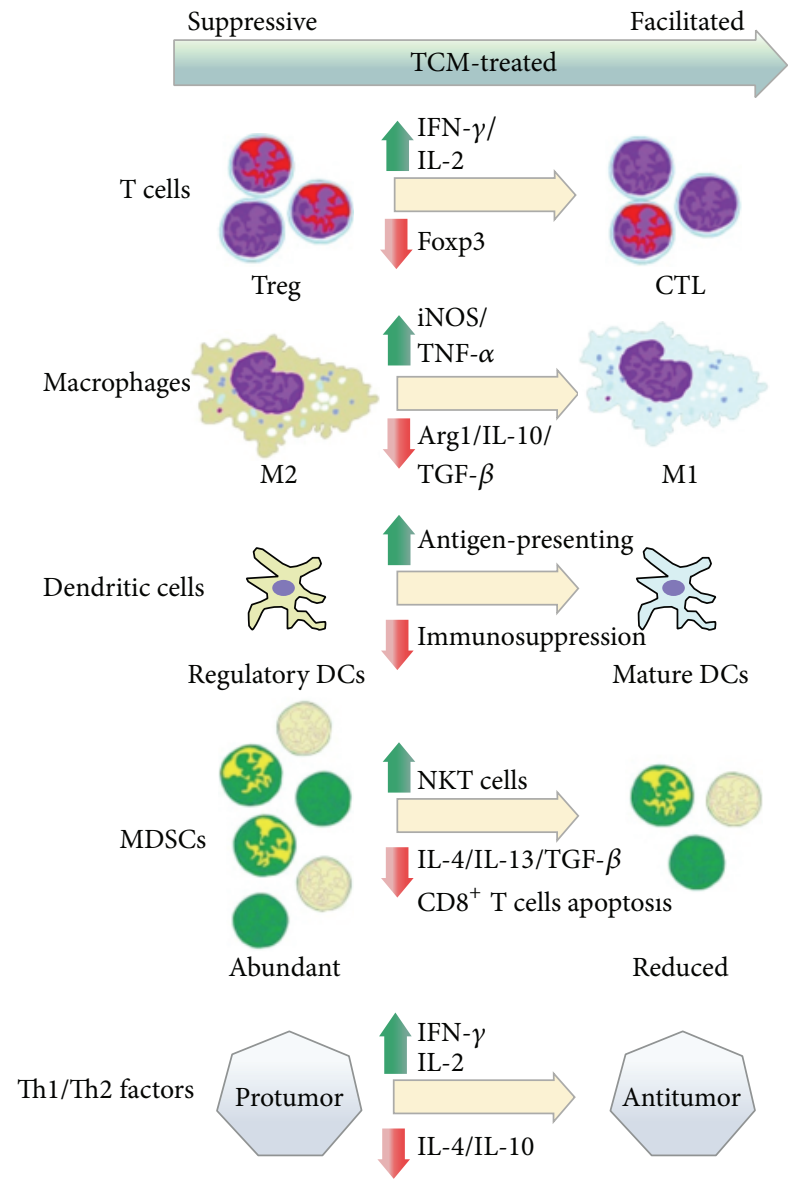

FIGURE 3: Molecular mechanism of TCM on regulating immune cells in TIM.

and Ganoderma) is an antitumor compound prescription of TCM, which has been proven effective in the clinical research [49]. Recent study in Lewis lung carcinoma bearing mice model showed FYN's effect on Tregs. It was found that the numbers of $\mathrm{CD} 4{ }^{+} \mathrm{CD} 25^{+}$regulatory T cells in spleen, thymus, and tumor were lower in the FYN group than in the model group $(P<0.05)$. The expression of Foxp3 mRNA in spleen, thymus, and tumor was also significantly downregulated in the FYN group [50]. There are other studies that showed main ingredients of FYN such as Astragaloside significantly increased IL-2 and IFN- $\gamma$ secretion of T cells and promoted T cells immune activity [51].

5.2. TCM Regulates M1/M2 Phenotypes of TAMs. Tumor associated macrophages (TAMs) derived from peripheral circulating monocytes occupy about $30 \%-50 \%$ of the total tumor stroma cells. After being recruited to the tumor microenvironment, TAMs differentiate into two polarized phenotypes, that is, the classic activation polarization (M1 phenotype) and the alternative activation polarization (M2 phenotype). Induced by IFN- $\gamma$, IL-6, and so forth, M1 macrophages display proinflammatory, antigen-presenting, and antitumor effects, through release of soluble enzyme,
TNF, and IFN and activation of T cell immune responses to inhibit tumor cells. However, M2 macrophages, activated by IL-10, IL-13, and so forth, have an immune regulating and suppressive role via multiple ways to promote tumor progression. Recent studies indicated TAMs tend to M2 protumor phenotype through stimulating tumor cell proliferation, inhibiting tumor immune microenvironment, promoting matrix remodeling, and accelerating angiogenesis and lymphangiogenesis $[52,53]$. Thus, TAMs are associated with tumor progression and metastasis.

Researchers reported TCM herbs could inhibit macrophages' inflammatory effect [54], and there are certain studies that showed TCM could switch the phenotype of TAMs from M2 to M1 during tumor progression. Schisandra polysaccharide (SCPP11) is an extract ingredient from herb medicine Schisandra chinensis, which has been used in TCM for centuries and proved to have antitumor activities. Recent study results showed that SCPP11 could increase the pinocytic activity of peritoneal macrophages in CTX-induced immunosuppression mice. Moreover, SCPP11 significantly increased immunoglobulin levels and cytokines levels in vivo and induced RAW264.7 cells (a monocyte/macrophage cell line of mice for in vitro experiments) to secrete cytokines in vitro and RAW264.7 cells pretreated with SCPP11 significantly inhibited the proliferation of HepG-2 cells, via promoting the expression of both iNOS protein and iNOS and TNF- $\alpha$ mRNA [55]. These results showed that SCPP11 could enhance the antitumor effect of macrophages. Other researchers found five extracts (UM01, QH11, BNQM, GNCC, and DCXC) of Cordyceps sinensis could significantly increase cell proliferation and NO production of RAW264.7 cells [56]. Zhang et al. found that Ganoderma atrum polysaccharide (PSG-1) increased macrophage phagocytosis and the levels of cytokines and nitride oxide through TLR4-mediated NF- $\kappa \mathrm{B}$ and MAPK signaling pathways in S180 tumor bearing mice model [57]. Shenqi Fuzheng Injection (Radix Codonopsis, Radix Astragali) was indicated enhancing peritoneal macrophage phagocytosis in immunosuppressed mice as well [58]. Our studies found similar results that TCM decoction Fuzheng Jiedu Formula (FZJD) (Radix Astragali, Radix Codonopsis, Rhizoma Atractylodis Macrocephalae, Radix Polygoni Multiflori, Fructus Lycii, Yunnan Manyleaf Paris Rhizome, Hedyotis diffusa, and Actinidia arguta Planch.), including Radix Codonopsis and Radix Astragali, not only promoted macrophage phagocytosis but also enhanced M1/M2 and anti-/protumor factors of TAMs in tumor microenvironment. Specifically, FZJD reduced IL-10 and TGF- $\beta$ expression and raised the ratio of iNOS/Arg1 which represents the M1/M2 proportion. All the findings above illustrate Chinese medicinal herbs could inhibit tumor cells by promoting macrophages antitumor function and reversing TAM phenotype M2 to M1.

5.3. TCM Enhancing Antigen-Presenting Function of Dendritic Cells (DCs). Dendritic cells (DCs) are professional antigenpresenting cells, which activate initial $\mathrm{T}$ cell by presenting and delivering antigens and mediate acquired tumor immune cytotoxicity. However, recent studies found a subset of DCs in 
the tumor microenvironment inducing immunosuppression called regulatory DCs or tumor associated DCs (TADCs). These TADCs have a low ability to present antigen, induce $\mathrm{T}$ cells differentiating to Treg subtype, and consequently impair T cell-mediated tumor killing effects. Furthermore, TADCs lead to a decreasing ratio of Th cells and effective $T$ cells apoptosis through a way such as reactive oxygen species, the indoleamine 2, 3-2 oxidase (IDO), and releasing immune suppressive factors [59]. TADCs are also involved in tumor angiogenesis, tumor cell proliferation, and invasion [60]. Thus, we can see from above that DCs have a similar polarization like macrophages (M1/M2). Regulating dendritic cells and enhancing their antigen-presenting function could be a possible way for TCM antitumor effects.

Zhang and Liu found that TCM with function of supplementing $\mathrm{Pi}$ and nourishing Shen could improve the inflammatory function of DCs in patients with chronic hepatitis B [61]. Our laboratory group explored the possible influence of TCM drug FLP on regulating DCs in peripheral blood, spleen, and tumor in mice with transplanted Lewis lung cancer. We found that the percentages of DCs (per thousand) in tumor bearing mice were increased to $2.55 \pm$ 0.29 in peripheral blood and $2.70 \pm 0.63$ in spleen $(P<$ 0.01) after FLP treatment [62]. Further studies showed FLP promoted DCs maturity, reversed DCs regulatory (immunosuppressive) phenotype, and increased DCs membrane $\mathrm{MHC}$ II, CD80, CD83, CD86, and CD40 expression. FLP also promoted the IL-12 secretion of DCs and enhanced the function of DC-LPAK tumor killing way [34, 63]. These results indicate TCM may have regulating effect on DCs.

5.4. TCM Restrains Myeloid Derived Suppressor Cells (MDSCs). Myeloid derived suppressor cells (MDSCs) are a special kind of cell population which play essential role in malignant tumor immune suppression. The main immune inhibition effects of MDSCs reflect on suppressing T cells related immune responses and inducing $T$ cell apoptosis, promoting inflammatory mediated tumor recurrence and metastasis. MDSCs are a group of heterogeneous cells, including immature macrophages, dendritic cells, and granulocytes. Pathological stimulations like malignant tumor and inflammation block immature bone marrow cells differentiation into mature cells, leading to the expansion of MDSCs and contributing to the negative regulation of tumor immune response. MDSCs facilitate angiogenesis by releasing VEGF, MMPs, TGF- $\beta$, and so forth and inhibiting T cell-mediated tumor acquired immune responses with overexpression of Arg1, iNOS, Ros, and so forth. The increasing IL-10 productivity of MDSCs restricts macrophages and DCs antitumor function as well [64].

Since 2007, we established the collaboration with the National Cancer Institute (NCI) Molecular Immunoregulation Laboratory and carried out series studies on the immune regulation effect of TCM. The results showed TCM herbs Radix Ginseng and Radix Notoginseng had a certain inhibitory effect on MDSCs phenotype and protumor functions [65]. Our recent studies found that the levels of MDSCs in breast cancer patient peripheral blood are positively associated with tumor progression and TCM decoction Shugan Jianpi Formula (Radix Bupleuri, Radix Paeoniae Alba, Spica Prunellae, Radix Curcumae, Holboellia Fargesii Reaub, Radix Astragali, Radix Notoginseng, and Radix Glycyrrhizae) (SGJP) had an inhibition function in MDSCs proliferation and could prevent MDSCs induced IL4, IL-13, and TGF- $\beta$ expression and $\mathrm{CD}^{+} \mathrm{T}$ cells apoptosis. Meanwhile, SGJP enhanced and regulated inflammatory functions of NKT cells, which were associated with MDSCs regulation [66]. These above were addressed by Jak-Stats signal pathways and indicated TCM herbs affecting MDSCs.

\subsection{TCM Regulates Th1/Th2 Immune Factors Secretion.} Th1/Th2 immune factors also have a polarization of promoting antitumor immunocompetence and tumorigenesis ability. Thl factors like IL-2, IL-6, TNF- $\alpha$, and INF- $\gamma$, are linked to proinflammation, cytotoxic and cytophagic enhancement, and other antitumor functions, while Th2 factors, for example, IL-4, IL-10, and TGF- $\beta$, play a suppressive role in tumor immune microenvironment and promote tumor recurrence and metastasis.

Wei et al. $[67,68]$ found that TCM herb Radix Astragali (AG) and tetramethylpyrazine (TTMP) extracted from a medicinal herb Ligusticum chuanxiong could reverse predominance of Th2 cytokines in lung cancer patients. AG enhanced the levels of Th1 cytokine (IFN- $\gamma$ and IL-2) as well as its transcript factor (T-bet) expression in culture supernatant and reduced those of Th2 cytokines in cultured peripheral blood mononuclear cells (PBMC) of lung cancer patients. TTMP could enhance supernatant concentration and gene expression levels of IFN- $\gamma$, IL-2, and T-bet but reduce those of type 2 cytokines (e.g., IL-4, IL-10). These results demonstrated that AG and TTMP could reverse the type 2 dominant status, which might offer an alternative therapeutic regime for lung cancer patients. Other studies of TCM compound decoctions, containing Astragalus composition, showed a similar result as well. Fuzheng Yiliu Decoction (FYD) (Ginseng, Astragalus, Ganoderma lucidum, Angelica sinensis, and Lycium chinense) remarkably inhibited proliferation and induced apoptosis of hepatoma cells at least by promoting the production of IL- 2 and TNF- $\alpha$ in vivo [69]. Wang and Chen [70] observed the effect of Aidi injection (Mylabris, Radix Ginseng, Radix Astragali, and Radix et Caulis Acanthopanacis Senticosi) on peripheral blood expression of Th1/Th2 transcription factors and cytokines in patients with esophageal squamous cell carcinoma (ESCC) during radiotherapy. They found a Th2 toward drift phenomenon that, compared with the healthy control group, the expressions of Th1 type transcription factor T-bet and cytokines IFN- $\gamma$, IL- 2 in ESCC patients were significantly lower, while expressions of Th2 type transcription factor GATA-3 and cytokines IL-4, IL-10 were significantly higher. When combined with intravenous dripping of Aidi injection during radiotherapy, the Th2 toward drift was inhibited. These results demonstrated that TCM herbs might reverse the Th2 predominant status, which is a probable alternative therapeutic regime in future. 
Suppressive phenotypes of immune cells are regulated and reversed by TCM treating. Concretely, inflammatory $\mathrm{T}$ cells and NKT cells increasingly proliferate in contrast to the reducing quantity of regulatory $\mathrm{T}$ cells and MDSCs. Suppressive macrophages and DCs change their functions to antitumor effects, such as M2 to M1 phenotype reversing and DCs maturation. All these alterations are influenced and accompanied by increasing inflammatory factors expression and immune suppressive cytokines decreasing.

\section{Difference between TCM and Modern Western Medicine in Cancer Immunotherapy}

6.1. TCM Has Multitarget and Bidirectional Immunoregulation Effect. Based on the preliminary studies, the multitarget function of TCM compound on regulating TIM is assigned to its multicomponents. First, the compound is usually composed of more than one herb, and the herb often consists of various ingredients and each ingredient always contains different kinds of chemicals. There are some certain chemicals extracted from TCM herbs that are found to have immune regulating function: specifically, Ganoderma lucidum polysaccharides (GLPS), a kind of effective ingredient extracted from Ganoderma lucidum (G. lucidum), which has long been prescribed to prevent and treat various human diseases, particularly in China, Japan, and Korea. Some researches showed that GLPS had a potential role in cancer treatment; further studies suggested that the antitumor activities of GLPS are mediated by its immunoregulation effect. GLPS might act on immune-related cells as well as immune cells such as B lymphocytes, T lymphocytes, dendritic cells, macrophages, and natural killer cells [71]. Solanum nigrum Linn., another effective TCM herb in cancer treatment, has been proven to have antitumor activity by enhancing the $\mathrm{CD}^{+} / \mathrm{CD}^{+}$ratio of the $\mathrm{T}$ lymphocyte subpopulation. Razali et al. [72] named SN-ppF3 from Solanum nigrum Linn. polysaccharide that could significantly induce phagocytosis activity and stimulated the production of TNF- $\alpha$ and IL- 6 and nitric oxide synthase expression of RAW264.7 cells.

Besides the ingredient found directly from the herbs, the postmetabolized ingredients of TCM compound may also play an immunoregulating role. Bae et al. [73] found that, after oral administration of BST204, a purified ginseng dry extract containing ginsenosides such as Rgl and Rh1, only the S epimers, S-Rh2 and S-Rg3, could be determined in rat plasma. Studies also found that the oral bioavailability of ginsenoside Rg1 is very low in rats and ginsenosides might be metabolized by intestinal microflora before absorption into blood $[74,75]$. These indicated that some ingredients in TCM herbs might be effective after being metabolized or some of the chemicals inactive in vitro could play roles in organisms after being metabolized. Continuing in depth studies, metabolite of ginsenosides compound $\mathrm{K}$ was found in suppressing the activation of the NF- $\kappa \mathrm{B}$ pathway and attenuating metastatic growth of hepatocellular carcinoma [76]. Another study showed that Rh2 metabolic modification ginsenoside aglycone protopanaxadiol (aPPD) activated in treating prostate cancer combined with docetaxel and was more effective than its prototype [77]. TCM herbs such as ginseng have been proved to have immune enhancement, and it is a possible hypothesis that their metabolites modulate immune microenvironments.

As mentioned above, due to its complicated ingredients TCM compound has a multitarget effect. It might act directly on various immune cells, while it can also play a role through regulating immune factors in different immune signal pathways [78]. Another important feature of TCM is its bidirectional effect. For instance, Radix Astragali has effects on both neoplasms (immune enhancement) and rheumatoid arthritis (immune suppression) [79, 80]. In addition, TIM brings out abnormal expression of signal pathways and cytokines; meanwhile the ratio of function-opposite immune cell phenotypes such as M1/M2 macrophages is also imbalanced in TIM, which is called disharmony according to TCM theory. TCM always affect multiple targets, and they could appropriately reverse an unbalanced state (immunologic derangement in TIM) to a relative harmony state.

6.2. Western Medicine Acts as Guided Missiles in Cancer Immunotherapy. The characteristic of Western medicine is focused on definite targets that means Western medicine has specific targets relatively visualized and detectable, just like a guided missile hit a target with a minimal error. These immunotherapies are guided into the following fields: (1) monoclonal antibodies against either tumor cells (bevacizumab [81], cixutumumab [82], rituximab [83], etc.) or immunosuppressive factors (tremelimumab [84], nivolumab [85], etc.); (2) tumor vaccines based on polypeptide (MAGEA3 [86], etc.), DCs (belagenpumatucel-L [87], etc.), or other targets vaccines; (3) immune cells adoptive treatment, such as CIK [88]; (4) soluble tumor virus gene therapy [89], such as GC0070 and GL-ONC1. These indicate the convinced evidences and targets of Western medicine immunotherapy, but there are some problems and side effects, such as the dermatologic events associated with cixutumumab [90].

To make up for deficiencies of Western medicine specificity, it is feasible to introduce TCM in modern immunotherapies, such as the immune enhancement and side effects remission [91, 92]. Above all, it is benefit to patients that we combine the Western medicine (specificity) with TCM (multitarget and bidirectional regulating) in immunotherapies, and there is a promising field waiting us to study and explore.

\section{Summary}

TCM plays an important role in cancer treatment in China via diversity ways (Figure 4). The formation and dynamic changes of TIM are a multifaceted process and are including multiple targets, different types of cells, and various signal pathways. Here we maintain that the TCM, both monomer and compound, has a certain effect on regulating TIM, and ways and points the TCM is affecting are diverse. More specifically, TCM herbs influenced not only tumor cells, but immune cells and cytokines as well as signal pathways 


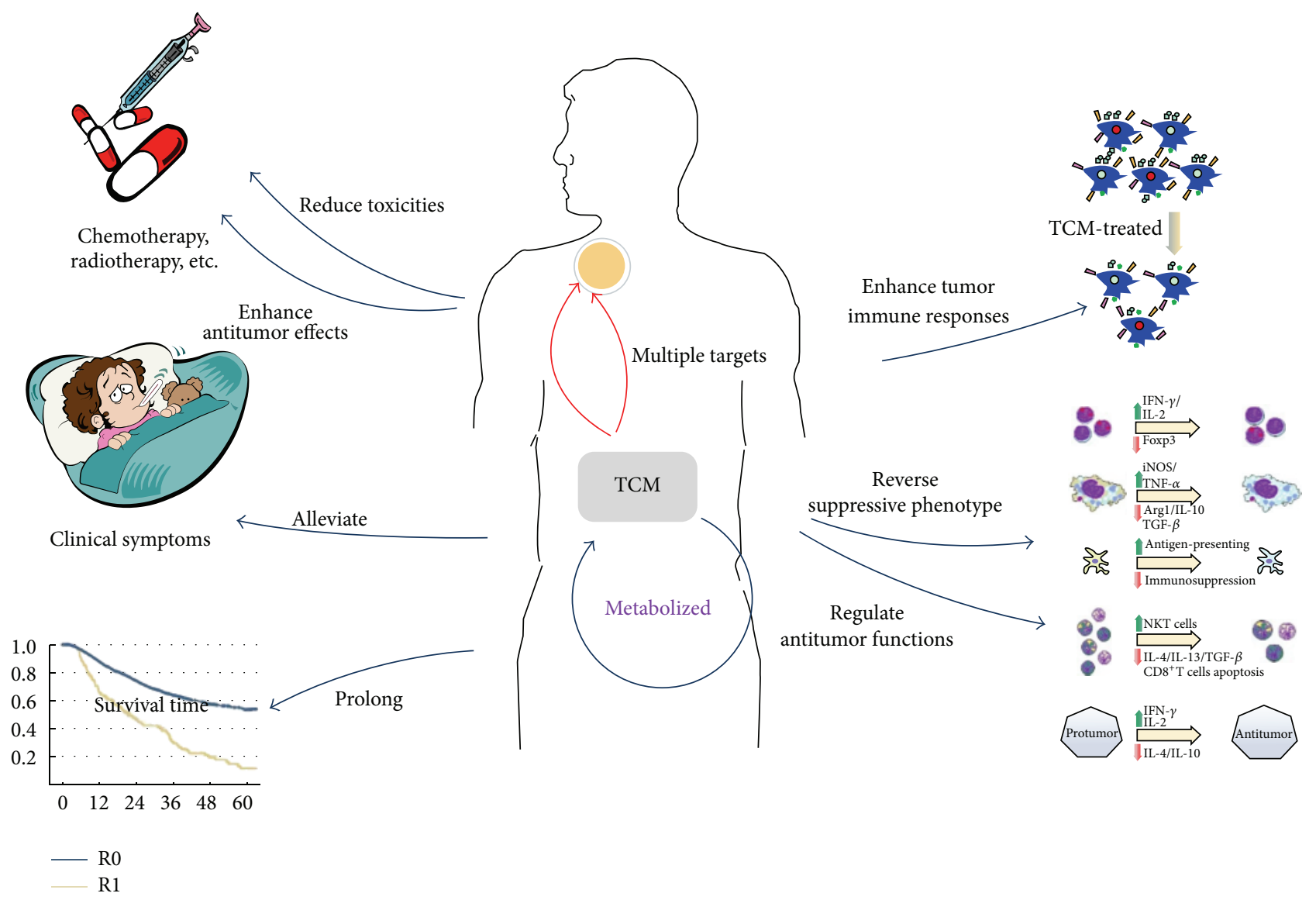

FIGURE 4: General functions and mechanisms of TCM on tumor therapies.

$[93,94]$. For the further analysis of these TCM herbs, we can see Radix Codonopsis, Radix Ginseng, Radix Astragali Preparata, Rhizoma Atractylodis Macrocephalae, Yunnan Manyleaf Paris Rhizome, Hedyotis diffusa, and so forth exist in kinds of TCM compound decoctions, which have different roles in regulating TIM; that is to say some of TCM herbs may have multiple effect on TIM. Treatment based on syndrome (ZHENG) differentiation is the characteristic and treatment guide of TCM. Studies showed tumor microenvironment differentiated under different TCM ZHENG models and had a relationship with treatment response to herbal medicine [95]. TCM herbs are cataloged according to their effect on certain TCM ZHENGs as Radix Codonopsis, Radix Ginseng, Radix Astragali Preparata, and Rhizoma Atractylodis Macrocephalae are tonified medicines to treat Qi deficiency ZHENG while Yunnan Manyleaf Paris Rhizome and Hedyotis diffusa relieve heat-toxin ZHENG in TCM pharmacology. TCM herbs are also considered to have dual-direction regulating effects on TIM as enhancing tumor cells antigenic responses and immune cells antitumor abilities while inhibiting the tumorigenesis functions. Above all, it is worth a further study to figure out the relationship and biochemical mechanism of different catalogs of TCM herbs in regulating TIM. With the rapid development of immunology, TCM effect on regulating TIM will play a more important role in tumor complementary and alternative therapies.
It has been accepted in China that TCM can reduce the toxicity and enhance the antitumor effect of chemotherapy and radiotherapy, alleviate tumor-induced clinical symptoms, and prolong the survival time of postoperational and advanced stage cancer patients [4]. Meanwhile, TCM regulates TIM by enhancing tumor immune responses, reverses suppressive phenotype of immune cells, and promotes their antitumor functions.
Abbreviations
ANG: Angiopoietin
APRIL: A proliferation inducing ligand
COX-2: Cyclooxygenase-2
CSC: Cancer stem-like cell
DC: Dendritic cell
EGF: Epidermal growth factor
EMT: Epithelial mesenchymal transition
Erk: $\quad$ Extracellular regulated protein kinases
HCC: Hepatocellular carcinoma
HGF: Hepatocyte growth factor
HLA: Human leukocyte antigen
IGF-1: Insulin-like growth factor-1
IL: Interleukin
INF- $\gamma$ : Gamma interferon
MDSC: Myeloid derived suppressor cell 
MHC: Major histocompatibility complex

MMP: Matrix metalloproteinase

TAM: Tumor associated macrophage

TCM: Traditional Chinese medicine

TGF- $\beta$ : Transforming growth factor-beta

TIM: Tumor immunosuppressive microenvironment

TNF- $\alpha$ : Tumor necrosis factor-alpha

VEGF: Vascular endothelial growth factor.

\section{Conflict of Interests}

The authors declare that there is no conflict of interests regarding the publication of this paper.

\section{Acknowledgment}

This work was supported by National Natural Science Foundation of China (nos. 81072802, 81273946, and 81473463).

\section{References}

[1] G. P. Dunn, A. T. Bruce, H. Ikeda, L. J. Old, and R. D. Schreiber, "Cancer immunoediting: from immunosurveillance to tumor escape," Nature Immunology, vol. 3, no. 11, pp. 991-998, 2002.

[2] G. P. Dunn, L. J. Old, and R. D. Schreiber, "The three Es of cancer immunoediting," Annual Review of Immunology, vol. 22, pp. 329-360, 2004.

[3] J. Li and H.-S. Lin, "Integrative medicine: a characteristic China model for cancer treatment," Chinese Journal of Integrative Medicine, vol. 17, no. 4, pp. 243-245, 2011.

[4] J. Li, L. Li, R. Liu, and H.-S. Lin, "Establishing Chinese medicine characteristic tumor response evaluation system is the key to promote internationalization of Chinese medicine oncology," Chinese Journal of Integrative Medicine, vol. 18, no. 10, pp. 730736, 2012.

[5] L. Xu, J.-M. Feng, J.-X. Li et al., “Tanshinone-1 induces tumor cell killing, enhanced by inhibition of secondary activation of signaling networks," Cell Death \& Disease, vol. 4, article e905, 2013.

[6] Y. Jia, Q. Guan, Y. Guo, and C. Du, "Reduction of inflammatory hyperplasia in the intestine in colon cancer-prone mice by water-extract of Cistanche deserticola," Phytotherapy Research, vol. 26, no. 6, pp. 812-819, 2012.

[7] M. Egeblad, E. S. Nakasone, and Z. Werb, “Tumors as organs: complex tissues that interface with the entire organism," Developmental Cell, vol. 18, no. 6, pp. 884-901, 2010.

[8] G. Kochan, D. Escors, K. Breckpot, and D. Guerrero-Setas, "Role of non-classical MHC class I molecules in cancer immunosuppression," OncoImmunology, vol. 2, no. 11, Article ID e26491, 2013.

[9] C. Fremd, F. Schuetz, C. Sohn, P. Beckhove, and C. Domschke, "B cell-regulated immune responses in tumor models and cancer patients," OncoImmunology, vol. 2, no. 7, Article ID e25443, 2013.

[10] D. Lindau, P. Gielen, M. Kroesen, P. Wesseling, and G. J. Adema, "The immunosuppressive tumour network: myeloid-derived suppressor cells, regulatory T cells and natural killer T cells," Immunology, vol. 138, no. 2, pp. 105-115, 2013.
[11] S. Ostrand-Rosenberg, P. Sinha, D. W. Beury, and V. K. Clements, "Cross-talk between myeloid-derived suppressor cells (MDSC), macrophages, and dendritic cells enhances tumor-induced immune suppression," Seminars in Cancer Biology, vol. 22, no. 4, pp. 275-281, 2012.

[12] G. Landskron, M. de La Fuente, P. Thuwajit, C. Thuwajit, and M. A. Hermoso, "Chronic inflammation and cytokines in the tumor microenvironment," Journal of Immunology Research, vol. 2014, Article ID 149185, 19 pages, 2014.

[13] H. W. Chung and J.-B. Lim, "Role of the tumor microenvironment in the pathogenesis of gastric carcinoma," World Journal of Gastroenterology, vol. 20, no. 7, pp. 1667-1680, 2014.

[14] H. Bolouri, "Network dynamics in the tumor microenvironment," Seminars in Cancer Biology, vol. 30, pp. 52-59, 2015.

[15] J. M. Fritz, L. D. Dwyer-Nield, and A. M. Malkinson, "Stimulation of neoplastic mouse lung cell proliferation by alveolar macrophage-derived, insulin-like growth factor-1 can be blocked by inhibiting MEK and PI3K activation," Molecular Cancer, vol. 10, article 1476, 2011.

[16] F. Munari, S. Lonardi, M. A. Cassatella et al., "Tumor-associated macrophages as major source of APRIL in gastric MALT lymphoma," Blood, vol. 117, no. 24, pp. 6612-6616, 2011.

[17] M. Yang, J. Chen, F. Su et al., "Microvesicles secreted by macrophages shuttle invasion-potentiating microRNAs into breast cancer cells," Molecular Cancer, vol. 10, article 117, 2011.

[18] S. E. J. Chambers, C. L. O’Neill, T. M. O’Doherty, R. J. Medina, and A. W. Stitt, "The role of immune-related myeloid cells in angiogenesis," Immunobiology, vol. 218, no. 11, pp. 1370-1375, 2013.

[19] B. Zhang, G. Yao, Y. Zhang, J. Gao, B. Yang, and Z. Rao, "M2Polarized tumor-associated macrophages are associated with poor prognoses resulting from accelerated lymphangiogenesis in lung adenocarcinoma," Clinics, vol. 66, no. 11, pp. 1879-1886, 2011.

[20] M. de Palma and L. Naldini, "Angiopoietin-2 TIEs up macrophages in tumor angiogenesis," Clinical Cancer Research, vol. 17, no. 16, pp. 5226-5232, 2011.

[21] S. Ran and K. E. Montgomery, "Macrophage-mediated lymphangiogenesis: the emerging role of macrophages as lymphatic endothelial progenitors," Cancers, vol. 4, no. 3, pp. 618-657, 2012.

[22] R. Guiet, E. van Goethem, C. Cougoule et al., "The process of macrophage migration promotes matrix metalloproteinaseindependent invasion by tumor cells," The Journal of Immunology, vol. 187, no. 7, pp. 3806-3814, 2011.

[23] T. Tsuji, S. Ibaragi, and G.-F. Hu, "Epithelial-mesenchymal transition and cell cooperativity in metastasis," Cancer Research, vol. 69, no. 18, pp. 7135-7139, 2009.

[24] C.-Y. Liu, J.-Y. Xu, X.-Y. Shi et al., "M2-polarized tumorassociated macrophages promoted epithelial-mesenchymal transition in pancreatic cancer cells, partially through TLR4/IL10 signaling pathway," Laboratory Investigation, vol. 93, no. 7, pp. 844-854, 2013.

[25] B. Toh, X. Wang, J. Keeble et al., "Mesenchymal transition and dissemination of cancer cells is driven by myeloid-derived suppressor cells infiltrating the primary tumor," PLoS Biology, vol. 9, no. 9, Article ID e1001162, 2011.

[26] F. Iovino, S. Meraviglia, M. Spina et al., "Immunotherapy targeting colon cancer stem cells," Immunotherapy, vol. 3, no. 1, pp. 97-106, 2011.

[27] C. P. Beier, P. Kumar, K. Meyer et al., "The cancer stem cell subtype determines immune infiltration of Glioblastoma," Stem Cells and Development, vol. 21, no. 15, pp. 2753-2761, 2012. 
[28] J. Yang, D. Liao, C. Chen et al., “Tumor-associated macrophages regulate murine breast cancer stem cells through a novel paracrine egfr/stat3/sox-2 signaling pathway," Stem Cells, vol. 31, no. 2, pp. 248-258, 2013.

[29] K.-F. He, L. Zhang, C.-F. Huang et al., "CD163+ tumorassociated macrophages correlated with poor prognosis and cancer stem cells in oral squamous cell carcinoma," BioMed Research International, vol. 2014, Article ID 838632, 9 pages, 2014.

[30] T. X. Cui, I. Kryczek, L. Zhao et al., "Myeloid-derived suppressor cells enhance stemness of cancer cells by inducing microRNA101 and suppressing the corepressor CTBP2," Immunity, vol. 39, no. 3, pp. 611-621, 2013.

[31] J. Bukur, S. Jasinski, and B. Seliger, "The role of classical and non-classical HLA class I antigens in human tumors," Seminars in Cancer Biology, vol. 22, no. 4, pp. 350-358, 2012.

[32] Y.-L. Li, B.-G. Sun, T. Xiang, Z.-X. Chen, and S.-J. Zhang, "Effect of invigorating spleen and detoxification decoction on MHCI/MHCII in spleen-deficiency liver cancer rats survival," Journal of Chinese Medicinal Materials, vol. 37, no. 3, pp. 456462, 2014

[33] Y.-C. Kuan, W.-T. Lee, C.-L. Hung, C. Yang, and F. Sheu, "Investigating the function of a novel protein from Anoectochilus formosanus which induced macrophage differentiation through TLR4-mediated NF- $\kappa$ B activation," International Immunopharmacology, vol. 14, no. 1, pp. 114-120, 2012.

[34] H. Zheng, B. Piao, H. Lin, L. Xiong, and B. Hua, "Influence of Feiliuping Extractum and its modified formulas on function of dendritic cells," Journal of Beijing University of Traditional Chinese Medicine, vol. 30, no. 8, pp. 525-528,549,577, 2007.

[35] M. Villa-Morales and J. Fernández-Piqueras, "Targeting the Fas/FasL signaling pathway in cancer therapy," Expert Opinion on Therapeutic Targets, vol. 16, no. 1, pp. 85-101, 2012.

[36] J. Li, G. Z. Sun, X. Qi, Y. X. Pei, H. F. Yan, and Y. H. He, "Effect of 'YangWeiKangLiu' granules on peripheral blood T-lymphocyte rDNA transcription activity and Fas expression in gastric cancer metastasis," Chinese Journal of Integrative Medicine, vol. 8, no. 4, pp. 253-255, 2002.

[37] J. Li, G.-Z. Sun, H.-S. Lin et al., "The herb medicine formula 'Yang Wei Kang Liu' improves the survival of late stage gastric cancer patients and induces the apoptosis of human gastric cancer cell line through Fas/Fas ligand and Bax/Bcl-2 pathways," International Immunopharmacology, vol. 8, no. 9, pp. 1196-1206, 2008.

[38] K. Li, Z. Dan, and Y.-Q. Nie, “Gastric cancer stem cells in gastric carcinogenesis, progression, prevention and treatment," World Journal of Gastroenterology, vol. 20, no. 18, pp. 5420-5426, 2014.

[39] F. Mannello, "Understanding breast cancer stem cell heterogeneity: time to move on to a new research paradigm," $B M C$ Medicine, vol. 11, article 169, 2013.

[40] T. Szafarowski and M. J. Szczepanski, "Cancer stem cells in head and neck squamous cell carcinoma," Otolaryngologia Polska, vol. 68, no. 3, pp. 105-111, 2014.

[41] J. Pasquier and A. Rafii, "Role of the microenvironment in ovarian cancer stem cell maintenance," BioMed Research International, vol. 2013, Article ID 630782, 10 pages, 2013.

[42] R. Xie, H. Long, and B. Zhu, "Cancer stem cell and tumor immunity," Immunological Journal, vol. 26, no. 10, pp. 909-912, 2010.

[43] Y. Chang, Y. Zhao, H. Zhan, X. Wei, T. Liu, and B. Zheng, "Bufalin inhibits the differentiation and proliferation of human osteosarcoma cell line hMG63-derived cancer stem cells," Tumor Biology, vol. 35, no. 2, pp. 1075-1082, 2014.

[44] T. Zhang, K. Wang, J. Zhang et al., "Huaier aqueous extract inhibits colorectal cancer stem cell growth partially via downregulation of the Wnt/ $\beta$-catenin pathway," Oncology Letters, vol. 5, no. 4, pp. 1171-1176, 2013.

[45] H. C. Pan, D. W. Lai, K. H. Lan et al., "Honokiol thwarts gastric tumor growth and peritoneal dissemination by inhibiting Tpl2 in an orthotopic model," Carcinogenesis, vol. 34, no. 11, pp. 25682579, 2013.

[46] S. Ponnurangam, J. M. V. Mammen, S. Ramalingam et al., "Honokiol in combination with radiation targets notch signaling to inhibit colon cancer stem cells," Molecular Cancer Therapeutics, vol. 11, no. 4, pp. 963-972, 2012.

[47] M. I. Carvalho, I. Pires, J. Prada, and F. L. Queiroga, "A role for T-lymphocytes in human breast cancer and in canine mammary tumors," BioMed Research International, vol. 2014, Article ID 130894, 11 pages, 2014.

[48] T. F. Gajewski, H. Schreiber, and Y.-X. Fu, "Innate and adaptive immune cells in the tumor microenvironment," Nature Immunology, vol. 14, no. 10, pp. 1014-1022, 2013.

[49] Y. Liu, "Antineoplastic synergistic party and lung YanNing party combined with chemotherapy treatment of advanced lung adenocarcinoma random parallel control study," Journal of Practical Traditional Chinese Internal Medicine, vol. 27, no. 6, pp. 111-113, 2013.

[50] J. Guo, J.-Y. Wang, Z. Zheng, Q. Wang, and C.-S. Dong, "Effects of chinese herbal medicine Feiyanning Decoction on the ratio of CD4+ CD25+ regulatory T cells and expression of transcription factor Foxp3 in mice bearing lewis lung carcinoma," Journal of Chinese Integrative Medicine, vol. 10, no. 5, pp. 584-590, 2012.

[51] C.-P. Wan, L.-X. Gao, L.-F. Hou et al., "Astragaloside II triggers $\mathrm{T}$ cell activation through regulation of CD45 protein tyrosine phosphatase activity," Acta Pharmacologica Sinica, vol. 34, no. 4, pp. 522-530, 2013.

[52] D. Capece, M. Fischietti, D. Verzella et al., "The inflammatory microenvironment in hepatocellular carcinoma: a pivotal role for tumor-associated macrophages," BioMed Research International, vol. 2013, Article ID 187204, 15 pages, 2013.

[53] Q.-J. Guo and J. Li, “The role of tumor-associated macrophages in remodeling tumor immune microenvironment," Tumor, vol. 33, no. 10, pp. 922-927, 2013.

[54] G. Chen, K.-K. Li, C.-H. Fung et al., "Er-Miao-San, a traditional herbal formula containing Rhizoma Atractylodis and Cortex Phellodendri inhibits inflammatory mediators in LPSstimulated RAW264.7 macrophages through inhibition of NF$\kappa \mathrm{B}$ pathway and MAPKs activation," Journal of Ethnopharmacology, vol. 154, no. 3, pp. 711-718, 2014.

[55] T. Zhao, Y. Feng, J. Li et al., "Schisandra polysaccharide evokes immunomodulatory activity through TLR 4-mediated activation of macrophages," International Journal of Biological Macromolecules, vol. 65, pp. 33-40, 2014.

[56] L.-Z. Meng, B.-Q. Lin, B. Wang et al., "Mycelia extracts of fungal strains isolated from Cordyceps sinensis differently enhance the function of RAW 264.7 macrophages," Journal of Ethnopharmacology, vol. 148, no. 3, pp. 818-825, 2013.

[57] S. Zhang, S. Nie, D. Huang, J. Huang, Y. Wang, and M. Xie, "Polysaccharide from Ganoderma atrum evokes antitumor activity via Toll-like receptor 4-mediated NF- $\kappa \mathrm{B}$ and mitogenactivated protein kinase signaling pathways," Journal of Agricultural and Food Chemistry, vol. 61, no. 15, pp. 3676-3682, 2013. 
[58] J. Wang, X. Tong, P. Li, H. Cao, and W. Su, "Immuno-enhancement effects of Shenqi Fuzheng Injection on cyclophosphamide-induced immunosuppression in Balb/c mice," Journal of Ethnopharmacology, vol. 139, no. 3, pp. 788-795, 2012.

[59] B. Seliger and C. Massa, "The dark side of dendritic cells: development and exploitation of tolerogenic activity that favor tumor outgrowth and immune escape," Frontiers in Immunology, vol. 4, article 419, 2013

[60] Y. Ma, G. V. Shurin, D. W. Gutkin, and M. R. Shurin, "Tumor associated regulatory dendritic cells," Seminars in Cancer Biology, vol. 22, no. 4, pp. 298-306, 2012.

[61] Y.-H. Zhang and Y.-H. Liu, "Influence of TCM therapy for supplementing $\mathrm{Pi}$ and nourishing Shen on dendritic cell function in patients with chronic hepatitis B treated by lamivudine," Chinese Journal of Integrative Medicine, vol. 15, no. 1, pp. 60-62, 2009.

[62] H.-G. Zheng, B.-K. Piao, and B.-J. Hua, "Effect of feiliuping ointment and its disassembled prescriptions on dendritic cell of mice with transplanted Lewis lung cancer," Zhongguo Zhong Xi Yi Jie He Za Zhi, vol. 30, no. 12, pp. 1288-1291, 2010.

[63] Y. M. Zhou, B. K. Piao, H. G. Zheng et al., "Influence on DC stimulating LPAK anti-tumor activity by feiliuping extract and its decomposed recipe," Cancer Research on Prevention and Treatment, vol. 40, no. 1, pp. 3-6, 2013.

[64] Y. S. Khaled, B. J. Ammori, and E. Elkord, "Myeloid-derived suppressor cells in cancer: recent progress and prospects," Immunology and Cell Biology, vol. 91, no. 8, pp. 493-502, 2013.

[65] J. Li, X. Chen, H. S. Lin, J. J. Oppenheim, and O. M. Z. Howard, "Inhibitory effect of Sheng Qi Formula (SQF) on Gr-1 ${ }^{+} \mathrm{CD}_{11 \mathrm{~b}}{ }^{+}$ myeloid immunosuppressor cells (MIC) in the $4 \mathrm{~T} 1$ murine mammary cancer model," AACR Annual Meeting Abstract, vol. 2008, article 5336, 9th AACR Annual Meeting, San Diego, Calif, USA, April 2008, 2008.

[66] M. Taniguchi, K. I. Seino, and T. Nakayama, "The NKT cell system: bridging innate and acquired immunity," Nature Immunology, vol. 4, no. 12, pp. 1164-1165, 2003.

[67] H. Wei, R. Sun, W. Xiao et al., "Traditional Chinese medicine Astragalus reverses predominance of Th2 cytokines and their up-stream transcript factors in lung cancer patients," Oncology Reports, vol. 10, no. 5, pp. 1507-1512, 2003.

[68] H. Wei, R. Sun, W. Xiao et al., "Type two cytokines predominance of human lung cancer and its reverse by traditional Chinese medicine TTMP," Cellular \& Molecular Immunology., vol. 1, no. 1, pp. 63-70, 2004.

[69] X. Z. Chen, Z. Y. Cao, L. M. Liao, Z. Z. Liu, and J. Du, “Application of serum pharmacology in evaluating the antitumor effect of FuzhengYiliu Decoction from Chinese medicine," Chinese Journal of Integrative Medicine, vol. 20, no. 6, pp. 450-455, 2014.

[70] Q. Wang and D. Y. Chen, "Effect of Aidi injection on peripheral blood expression of Th1/Th2 transcription factors and cytokines in patients with esophageal squamous cell carcinoma during radiotherapy," Zhongguo Zhong Xi Yi Jie He ZaZhi, vol. 29, no. 5, pp. 394-397, 2009.

[71] Z. Xu, X. Chen, Z. Zhong, L. Chen, and Y. Wang, "Ganoderma lucidum polysaccharides: immunomodulation and potential anti-tumor activities," The American Journal of Chinese Medicine, vol. 39, no. 1, pp. 15-27, 2011.

[72] F. N. Razali, A. Ismail, N. Z. Abidin, and A. S. Shuib, "Stimulatory effects of polysaccharide fraction from Solanum nigrum on RAW 264.7 murine macrophage cells," PLoS ONE, vol. 9, no. 10, Article ID e108988, 2014.

[73] S. H. Bae, J. B. Park, Y. F. Zheng et al., "Pharmacokinetics and tissue distribution of ginsenoside Rh2 and Rg3 epimers after oral administration of BST204, a purified ginseng dry extract, in rats," Xenobiotica, vol. 44, no. 12, pp. 1099-1107, 2014.

[74] Z. Y. Tan, W. N. Xiong, X. Z. Huang, and J. Q. Liang, "Pharmacokinetics and bioavailability of ginsenoside Rg1 in rats," Zhong Yao Cai, vol. 36, no. 7, pp. 1121-1123, 2013.

[75] D. Peng, H. Wang, C. Qu, L. Xie, S. M. Wicks, and J. Xie, "Ginsenoside Re: its chemistry, metabolism and pharmacokinetics," Chinese Medicine, vol. 7, article 2, 2012.

[76] Y. Ming, Z. Chen, L. Chen et al., "Ginsenoside compound $\mathrm{K}$ attenuates metastatic growth of hepatocellular carcinoma, which is associated with the translocation of nuclear factor$\kappa \mathrm{B}$ p65 and reduction of matrix metalloproteinase-2/9," Planta Medica, vol. 77, no. 5, pp. 428-433, 2011.

[77] A. G. Musende, A. Eberding, W. Jia, E. Ramsay, M. B. Bally, and E. T. Guns, "Rh2 or its aglycone aPPD in combination with docetaxel for treatment of prostate cancer," Prostate, vol. 70, no. 13, pp. 1437-1447, 2010.

[78] W. Huihui, C. Yulong, and M. Junhua, "Molecular mechanism of immunity regulated by TCM in tumor patients," China Journal of Chinese Medicine, vol. 29, no. 197, pp. 1405-1408, 2014.

[79] B. Wang and M. Z. Chen, "Astragaloside IV possesses antiarthritic effect by preventing interleukin $1 \beta$-induced joint inflammation and cartilage damage," Archives of Pharmacal Research, vol. 37, no. 6, pp. 793-802, 2014.

[80] S. Sun, K. Zheng, H. Zhao et al., "Regulatory effect of astragalus polysaccharides on intestinal intraepithelial $\gamma \delta \mathrm{T}$ cells of tumor bearing mice," Molecules, vol. 19, no. 9, pp. 15224-15236, 2014.

[81] M. R. Stockler, F. Hilpert, M. Friedlander et al., "Patientreported outcome results from the open-label phase III AURELIA trial evaluating bevacizumab-containing therapy for platinum-resistant ovarian cancer," Journal of Clinical Oncology, vol. 32, no. 13, pp. 1309-1316, 2014.

[82] S. Malempati, B. Weigel, A. M. Ingle et al., "Phase I/II trial and pharmacokinetic study of cixutumumab in pediatric patients with refractory solid tumors and Ewing sarcoma: a report from the Children's Oncology Group," Journal of Clinical Oncology, vol. 30, no. 3, pp. 256-262, 2012.

[83] T. J. Molina, D. Canioni, C. Copie-Bergman et al., "Young patients with non-germinal center B-cell-like diffuse large Bcell lymphoma benefit from intensified chemotherapy with ACVBP plus rituximab compared with CHOP plus rituximab: analysis of data from the Groupe d'Etudes des Lymphomes de l'Adulte/Lymphoma Study Association phase III trial LNH 032B," Journal of Clinical Oncology, vol. 32, no. 35, pp. 3996-4003, 2014.

[84] A. Ribas, R. Kefford, M. A. Marshall et al., "Phase III randomized clinical trial comparing tremelimumab with standardof-care chemotherapy in patients with advanced melanoma," Journal of Clinical Oncology, vol. 31, no. 5, pp. 616-622, 2013.

[85] S. L. Topalian, M. Sznol, D. F. McDermott et al., "Survival, durable tumor remission, and long-term safety in patients with advanced melanoma receiving nivolumab," Journal of Clinical Oncology, vol. 32, no. 10, pp. 1020-1030, 2014.

[86] J. Vansteenkiste, M. Zielinski, A. Linder et al., "Adjuvant MAGE-A3 immunotherapy in resected non-small-cell lung cancer: phase II randomized study results," Journal of Clinical Oncology, vol. 31, no. 19, pp. 2396-2403, 2013.

[87] J. R. Brahmer, "Harnessing the immune system for the treatment of non-small-cell lung cancer," Journal of Clinical Oncology, vol. 31, no. 8, pp. 1021-1028, 2013.

[88] X. Yu, H. Zhao, L. Liu et al., "A randomized phase II study of autologous cytokine-induced killer cells in treatment of 
hepatocelluar carcinoma," Journal of Clinical Immunology, vol. 34, no. 2, pp. 194-203, 2014.

[89] M. J. Atherton and B. D. Lichty, "Evolution of oncolytic viruses: novel strategies for cancer treatment," Immunotherapy, vol. 5, no. 11, pp. 1191-1206, 2013.

[90] M. Daher, M. E. Lacouture, D. Rathkopf et al., "Case series of dermatologic events associated with the insulin-like growth factor receptor 1 inhibitor cixutumumab," Journal of Clinical Oncology, vol. 29, no. 21, pp. e638-e640, 2011.

[91] M. Liu, H. Tan, X. Zhang et al., "Hematopoietic effects and mechanisms of Fufang E'jiao Jiang on radiotherapy and chemotherapy-induced myelosuppressed mice," Journal of Ethnopharmacology, vol. 152, no. 3, pp. 575-584, 2014.

[92] C. Zhao, J. Chen, B. Yu et al., "Effect of modified Taohongsiwu decoction on patients with chemotherapy-induced hand-foot syndrome," Journal of Traditional Chinese Medicine, vol. 34, no. 1, pp. 10-14, 2014.

[93] B. C. He, J. L. Gao, B. Q. Zhang et al., “Tetrandrine inhibits Wnt $/ \beta$-catenin signaling and suppresses tumor growth of human colorectal cancer," Molecular Pharmacology, vol. 79, no. 2, pp. 211-219, 2011.

[94] W. C. S. Cho and K. N. Leung, "In vitro and in vivo anti-tumor effects of Astragalus membranaceus," Cancer Letters, vol. 252, no. 1, pp. 43-54, 2007.

[95] Z. Chen, L. Y. Chen, P. Wang, H. Y. Dai, S. Gao, and K. Wang, "Tumor microenvironment varies under different TCM ZHENG models and correlates with treatment response to herbal medicine," Evidence-Based Complementary and Alternative Medicine, vol. 2012, Article ID 635702, 10 pages, 2012. 


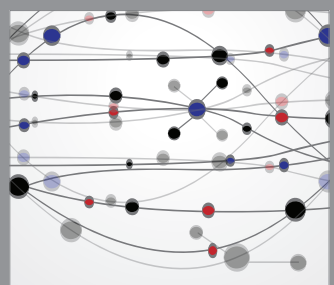

The Scientific World Journal
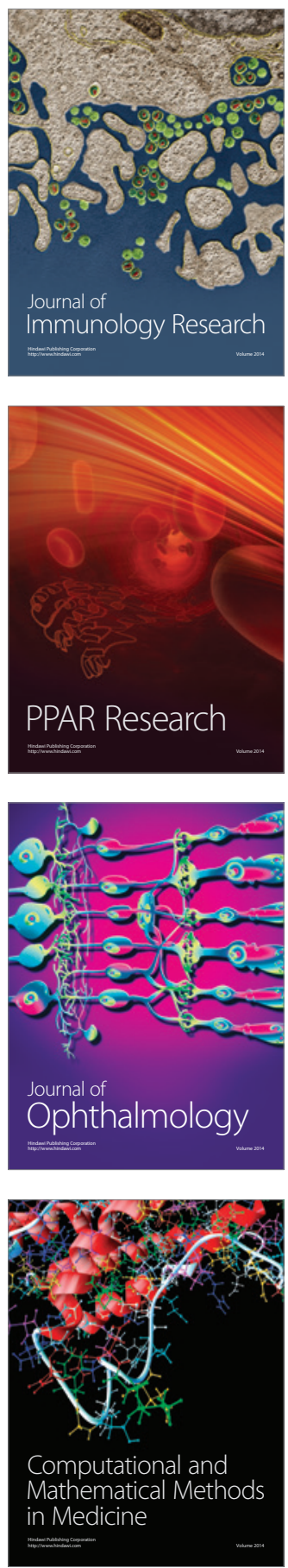

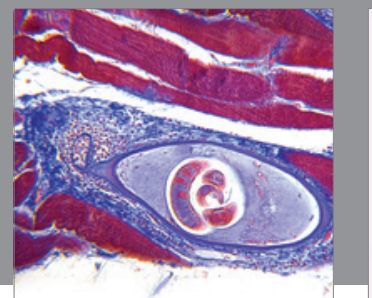

Gastroenterology

Research and Practice
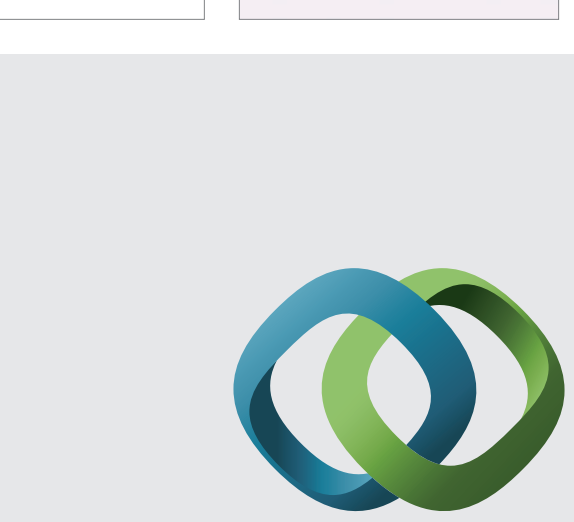

\section{Hindawi}

Submit your manuscripts at

http://www.hindawi.com
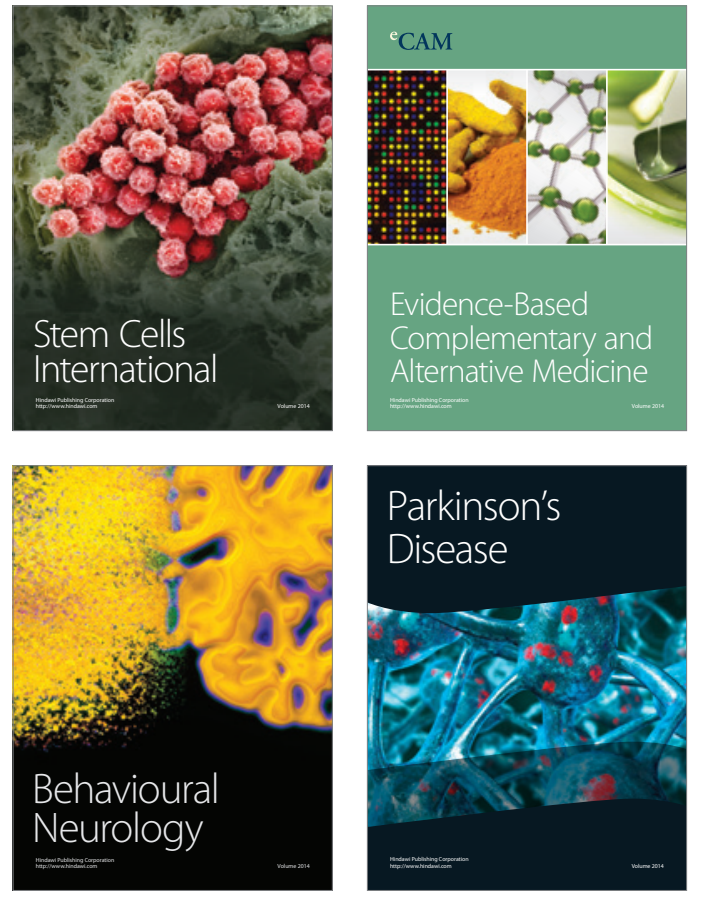
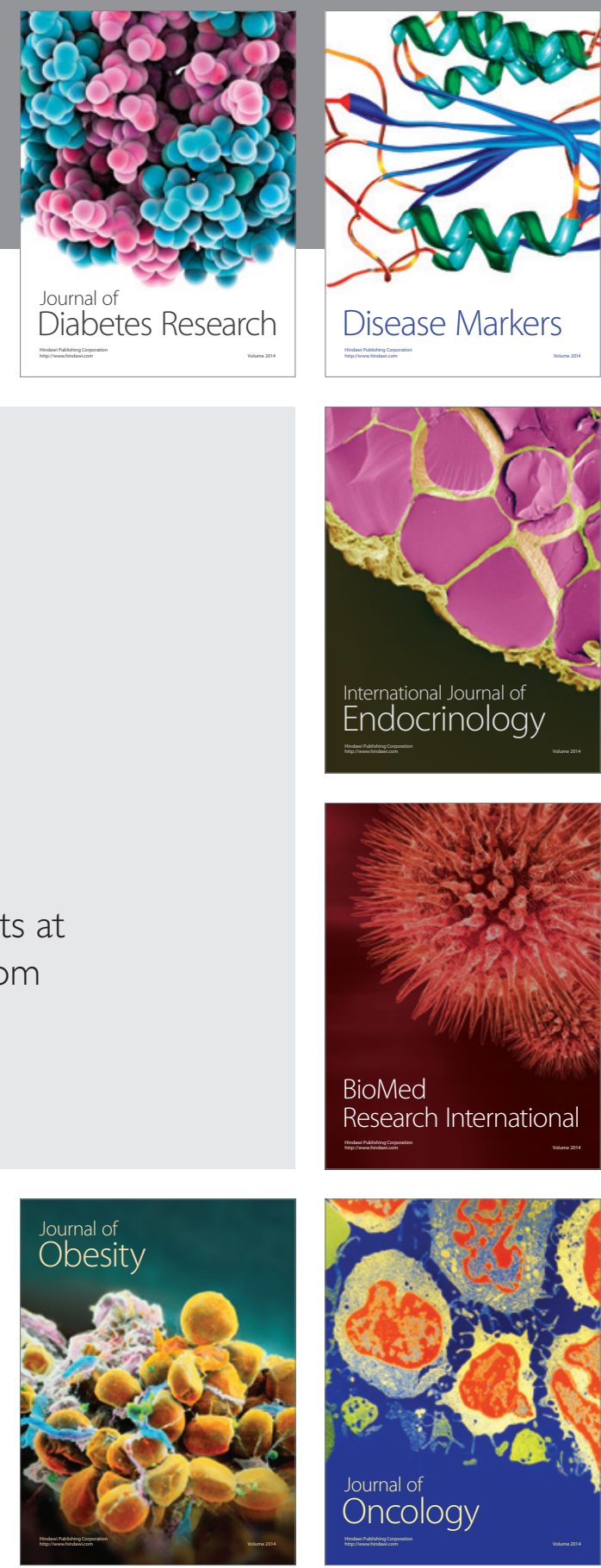

Disease Markers
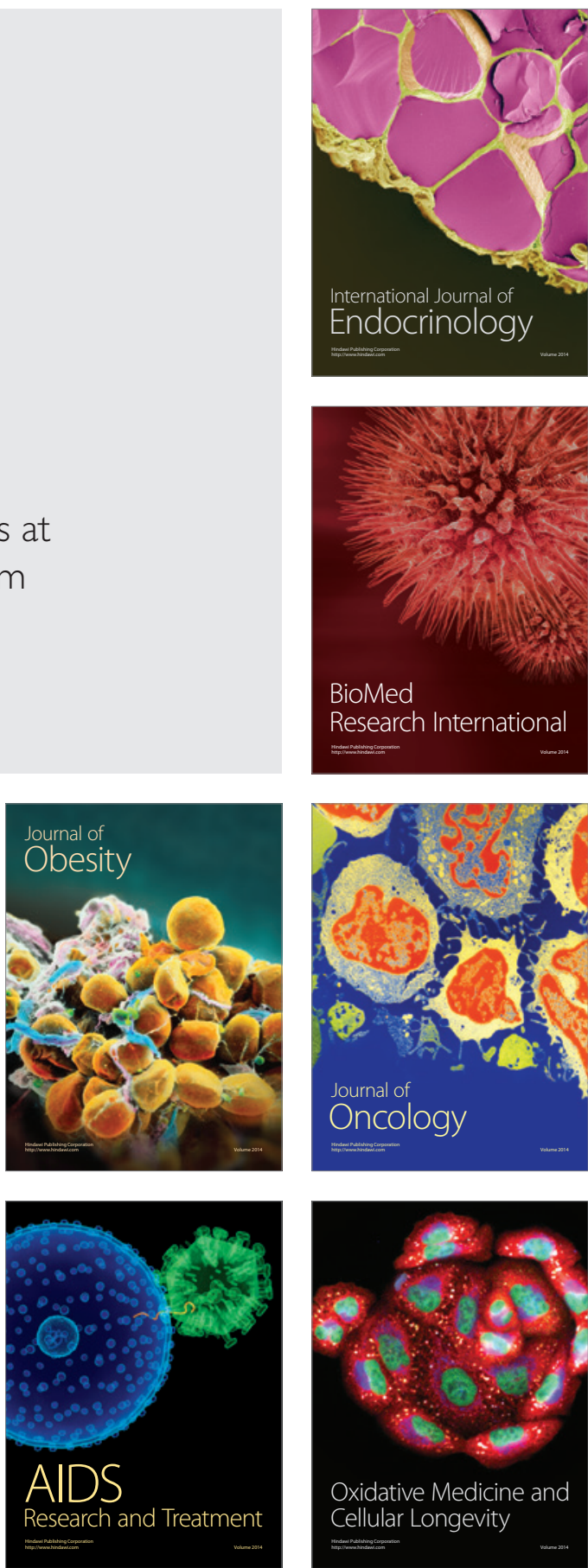